Published in final edited form as:

J Phys Act Health. 2016 June ; 13(6): 594-598. doi:10.1123/jpah.2015-0173.

\title{
Effects of a DVD-Delivered Exercise Intervention on Maintenance of Physical Activity in Older Adults
}

\author{
Jason Fanning ${ }^{1}$, Elizabeth A. Awick ${ }^{1}$, Thomas Wojcicki ${ }^{2}$, Neha Gothe ${ }^{3}$, Sarah Roberts ${ }^{1}$, \\ Diane K. Ehlers ${ }^{1}$, Robert Motl ${ }^{1}$, and Edward McAuley ${ }^{1}$ \\ ${ }^{1}$ Department of Kinesiology and Community Health, University of Illinois at Urbana-Champaign \\ ${ }^{2}$ Lansing School of Nursing \& Health Sciences, Bellarmine University \\ ${ }^{3}$ College of Education, Wayne State University
}

\section{Abstract}

Background-Previous research supports the efficacy of a 6-month DVD-delivered program for enhancing physical activity (PA) in older adults. In the present study, we examined the degree to which intervention-related increases in PA were maintained after a 6 month, no-contact follow up.

Methods-Follow-up assessments of PA via accelerometry and the Godin Leisure-Time Exercise Questionnaire (GLTEQ) were collected in a sample of older adults $(N=238)$. Repeated measures analyses of variance were conducted to examine changes in PA over the course of the follow-up period.

Results-For accelerometer measured PA, there was a significant time $\times$ treatment $\times$ age group interaction, $\mathrm{F}(1,203)=11.319, p=.001, \eta^{2}=.053$, such that younger ( $₫ 70$ years) intervention participants maintained high levels of PA across the follow-up period, while PA in older intervention and young control participants declined significantly. Rates of PA in older control participants remained low over the course of the follow-up period. Analyses of GLTEQ scores revealed similar, though less significant patterns.

Conclusions-DVD-based exercise programs may be effective for maintaining PA in younger members of the older adult population, however there remains a need to develop better strategies for promoting PA maintenance in older individuals when using home-based designs.

\section{Keywords}

Aging; Interventions; Physical Activity; Health Behavior

\section{Introduction}

Regular physical activity is necessary for maintenance of health and independent living as we age $\mathrm{e}^{1,2}$. Failure to engage in sufficient levels of activity is associated with disease states such as cancer, type II diabetes, osteoporosis, and depression, among others ${ }^{3}$. Low-active individuals also experience more rapid declines in physical function, substantially impacting overall quality of life and reducing the ability to live independently ${ }^{4-6}$. Unfortunately, national survey data suggest that in 2013 only $16 \%$ of those aged 65 and older met minimum 
physical activity guidelines ${ }^{7}$, while objective measurements place this number as low as $8.5 \%$ for those aged $60-69$ years and $6.3 \%$ for those aged 70 years and older ${ }^{8}$.

In an effort to increase physical activity levels, researchers have begun to utilize contemporary technologies to deliver physical activity interventions in the home. Doing so provides the ability to reach and engage a broad segment of the population, including those who are at risk of isolation and with reduced access to health care (e.g., those in rural settings, those with low physical functioning $)^{9,10}$. The digital video disk (DVD) is one such technology that holds promise for large-scale intervention delivery. DVDs can be easily distributed and DVD-players are inexpensive, widely used, and relatively simple to install and operate. A recent report suggested that a 6-month DVD-delivered physical activity program was effective for enhancing both physical function and quality of life in older adults, and that these effects were maintained at 6-months post-intervention ${ }^{5,11}$.

Additionally, the intervention was effective for increasing physical activity and this effect was more pronounced in an older cohort of study participants ${ }^{12}$. These results suggest the DVD is an efficacious platform for the delivery of intervention content to the older adult population. It is not known, however, how effective such programs are in maintaining physical activity in the absence of intervention support (e.g., contact with research staff, individualized feedback on progress).

The objective of the present study was to determine whether previously reported intervention-related increases in physical activity elicited via DVD-based physical activity program ${ }^{12}$ were maintained over a 6 -month follow-up period. We hypothesized those who received the intervention DVD would remain active upon cessation of the program. Given the broad range of ages in the study population (i.e., 64-95 years), and given that older participants experienced greater increases in activity during the intervention period ${ }^{12}$, we were also interested in determining whether maintenance of physical activity varied between younger and older study participants (i.e., those aged 70 or less as compared to those greater than 70 years of age).

\section{Methods}

\section{Study Design and Interventions}

The full study design and protocol have been detailed elsewhere ${ }^{10,11}$. Briefly, communitydwelling older adults were recruited from a 5,000 square mile region of central Illinois to participate in an exercise study examining the effects of a home-based exercise program on physical activity, functional limitations, and quality of life. Eligible participants $(\mathrm{N}=307)$ were randomized into one of two arms: the flexibility, toning, and balance (i.e., FlexToBa ${ }^{T M}$ ) intervention or an active aging DVD control condition.

Individuals randomized into the exercise condition received six exercise sessions delivered via DVD, and were encouraged to exercise with each session at least three times per week for four weeks. The exercise sessions were presented in sequential order, progressing in both complexity and difficulty across the six-month period. Participants in the control condition received Dr. Andrew Weil's commercially available "Healthy Aging" DVD, which focuses on various domains of healthy aging, including diet, exercise, sleep, and emotional well- 
being. Throughout the course of the intervention, both conditions received regularly scheduled telephone support calls from the research staff, administered with the same frequency and duration. All participants completed physical activity measures at baseline (i.e., month 0), at the end of the intervention (i.e., month 6), and at the end of a 6 month follow-up period (i.e., month 12).

\section{Measures}

Physical activity was assessed objectively using Actigraph brand accelerometers (Actigraph, Pensacola, FL: model GT1M or GT3X). Each participant was instructed to wear the accelerometer on the non-dominant hip for seven consecutive days, and record the time that they wore the device each day on a log. When scored with an interruption period of 30 minutes, those with 10 valid hours of wear time on at least three days were retained in analyses ${ }^{13}$. These data were downloaded as activity counts, which represent raw accelerations that have been summed over a specific epoch length (e.g., 60 seconds), and these counts vary based on frequency and intensity of the recorded acceleration ${ }^{14}$. Next, these data were processed in MeterPlus version 4.3 (Santech Incorporated, San Diego, CA) using older adult-specific cut-points developed by Copeland and Esliger ${ }^{15}$. The cut-point defining moderate to vigorous physical activity (MVPA) was $\geq 1,041$ counts per minute. Herein, we report total weekly minutes of MVPA to remain consistent with previously published results ${ }^{12}$. All analyses were also verified against average daily minutes of MVPA (thus reducing the influence of device wear time), and results did not differ.

Self-reported physical activity was obtained using the Godin Leisure-Time Exercise Questionnaire (GLTEQ) ${ }^{16}$. Participants reported the number of times each week they typically participate in strenuous, moderate, and mild exercise for at least 15 consecutive minutes during their free time. The frequency for each level of intensity is then multiplied by its MET value (i.e., 9, 5, and 3 METs, respectively), and summed to obtain a weekly leisure time physical activity score.

\section{Data Analyses}

Data from participants with valid physical activity data at baseline, end of intervention, and follow-up were analyzed, and extreme outliers were removed (acclerometer N=6; GLTEQ $\mathrm{N}=7$ ). In total, $32 \%$ of accelerometer-measured MVPA data were missing (remaining $\mathrm{N}=209$ ), and $25 \%$ of GLTEQ data were missing (remaining $\mathrm{N}=230$ ). Independent samples ttests were conducted to determine whether there were differences between individuals who completed physical activity assessments at follow-up and those who did not. Repeated measures analyses of variance were used to examine whether the rates of change varied significantly between conditions for accelerometer-measured MVPA and GLTEQ score over the course of the follow-up period when controlling for baseline scores and gender. A bivariate age variable was created based on median age (i.e., those less than or equal to 70 years, and those greater than 70 years) and included as a fixed factor. Significant interactions were decomposed via Bonferoni-corrected post-hoc analyses. All data analyses were conducted in SPSS version 22 (Armonk, NY: IBM Corp). 


\section{Results}

\section{Demographic Characteristics}

There were no significant differences in baseline demographics between those with and without sufficient data (all $p s \geq 07)$, with the exception of education status $(p<.01)$ and age $(\mathrm{p}=.02)$. Those who were retained were more likely to have received a college degree and were younger than were those without sufficient valid data.

\section{Accelerometry}

The repeated measures analysis of variance revealed a non-significant treatment effect, $\mathrm{F}(1,203)=2.236, p=.14, \eta^{2}=.011$, that was in the expected direction: the FlexToBaTM condition obtained 21.10 additional minutes of MVPA at follow-up when compared with the control condition. No time $\times$ treatment interaction was observed, $\mathrm{F}(1,203)=.728, p=.40, \eta^{2}=$. 004, however the time $\times$ treatment $\times$ age group interaction was significant, $F(1,203)=11.319$, $p<.01, \eta^{2}=.053$. Post-hoc analyses indicated that when controlling for gender and baseline values, the younger FlexToBa ${ }^{T M}$ participants maintained elevated levels of physical activity across the follow-up period (an increase of 3.19 minutes of MVPA per week, $p=.83$ ), but both older FlexToBa ${ }^{T M}$ and younger control participants demonstrated significant decreases from month 6 to month 12 (a decrease of 46.14 minutes of MVPA per week, $p=.02$; and 38.25 minutes of MVPA per week, $p=.02$, respectively). The older control cohort did not substantially change physical activity levels across the follow-up period $(p=.15)$.

\section{Godin Leisure-Time Exercise Questionnaire}

The repeated measures analysis of variance for the GLTEQ showed a non-significant treatment effect that favored the FlexToBa ${ }^{T M}$ condition, $\mathrm{F}(1,224)=3.322, p=.07, \eta^{2}=.015$, reflecting a difference of 3.27 points between conditions at follow-up. The time $\times$ treatment effect was significant, $\mathrm{F}(1,224)=4.026, p=.05, \eta^{2}=.018$. Post-hoc analyses revealed that when controlling for gender and baseline GLTEQ score, there was a significant decrease of 3.85 points in the FlexToBa ${ }^{T M}$ condition over the follow-up period ( $\left.p=.04\right)$, while control participants reported a non-significant increase of 1.21 points $(p=.47)$. The time $\times$ treatment $\times$ age group interaction was not significant, $\mathrm{F}(1,224)=0.772, p=.38, \eta^{2}=.003$; however, the patterns observed were similar to those seen in accelerometer-measured MVPA. Table 2 displays unadjusted values for physical activity variables at each time point, and Table 3 displays physical activity variables at month 6 and month 12 when controlling for gender and baseline values.

\section{Discussion}

The purpose of this study was to determine whether intervention-related increases in physical activity would be maintained during a no-contact follow-up period. The FlexToBa ${ }^{T M}$ program was partially successful in this regard. Specifically, accelerometermeasured physical activity was maintained in the younger study participants (i.e., those 70 years of age and younger). When compared to control individuals in the same age cohort, young FlexToBa ${ }^{T M}$ participants achieved approximately 49 minutes of additional moderate to vigorous physical activity each week by the end of the follow-up. The older FlexToBa ${ }^{T M}$ 
participants, however, did not maintain the intervention-related increases in physical activity reported by Gothe et al. ${ }^{12}$, returning to levels below those observed at baseline.

By taking advantage of widespread use of technologies such as the DVD, broad dissemination of home-based interventions becomes more feasible, increasing their publichealth impact. Still, we know little about how such designs are received by individuals across the lifespan and with varied demographics. The results of this study highlight the unique challenges faced by researchers implementing home-based designs. Our findings suggest that perhaps among younger members of the older adult age group, a DVD-based exercise program such as the one utilized in this study is sufficient to promote long-term maintenance of physical activity; however older participants who responded positively to the structured nature of the intervention may not maintain their new physically active lifestyle as easily in its absence.

Although qualitative data assessing potential contributors to these differing responses were not collected in the present study, it is possible that removal of the social support features (i.e., telephone calls and feedback) was associated with reductions in activity levels. Previous research suggests that as individuals grow older, support from family, friends, and program staff become increasingly important ${ }^{17}$. Moreover, type of social support desired may differ depending on the phase of the physical activity regimen ${ }^{18}$. During the FlexToBa ${ }^{T M}$ intervention, regular one-on-one brief interactions were provided in a titrated manner throughout the intervention period via telephone support calls ${ }^{10,11}$. As noted previously, this period corresponded with a significant increase in levels of physical activity among older participants ${ }^{12}$, while the decrease in activity during the follow-up period coincided with cessation of researcher support. Future research utilizing a home-based design such as the one employed in the FlexToBa ${ }^{T M}$ study may benefit by including social support measures to determine whether different subgroups of older adults have different preferences and needs when striving to maintain physical activity in the long-term. Effectiveness trials, which often compare an intervention as delivered as an efficacy trial to one delivered in real-world settings ${ }^{19}$, may be an efficient means of investigating these questions. As older adults become increasingly receptive to contemporary technologies 20 , further research utilizing features such as text message and push notification prompting ${ }^{21}$, interactive voice response ${ }^{22}$, and real-time advice and graphical feedback ${ }^{23}$ as a means of providing social support is warranted.

Another important consideration is the nature of physical activity captured via activity monitor. The devices used in this study best capture ambulatory activity such as walking and stair climbing. Therefore, these data may suggest that those in the younger cohort were more likely to have adopted and maintained additional physical activity beyond the DVD-based program than were the older cohort. Although the FlexToBa ${ }^{T M}$ program does not directly integrate aerobic activity, its introductory DVD and handbook emphasize the importance of lifestyle and structured aerobic physical activities. In order to better investigate the extent to which study participants maintain engagement with study materials and incorporate additional activities, future work may benefit by allowing participants to record physical activities during non-contact periods. 
This study has several limitations. As previously noted ${ }^{11,12}$, the majority of the sample was female and Caucasian; however women outnumber men in the older adult population and are more likely to engage in health-related endeavors ${ }^{24}$, therefore this sample is likely representative of the older adult population as a whole. Additionally, those that provided follow-up data were younger and more likely to have a college education than were those who did not provide data. This does have potential implications for both physical activity behavior and the use of the program, as those who are younger or who have a higher level of education have been shown to be more likely to engage in physical activity ${ }^{25}$ and to adopt new technology ${ }^{20}$. It will be important for future work to establish the long-term efficacy of a DVD-based physical activity program in minority populations and in those with lower education levels. Finally, a greater number of control participants were included in analyses. Participants in this condition received the exercise DVDs upon completion of month 12 assessments, perhaps incentivizing completion of testing materials. A challenging task for future work is to continue to develop strategies to retain participants during extended followup periods.

In spite of these limitations, this work provides an early examination of the long-term effects of a DVD-based exercise program for increasing and maintaining physical activity in older adults. It highlights the need to examine subgroups within the older adult population, as this age group encompasses a wide array of ages and associated needs and preferences.

\section{Conclusion}

In summary, this study provides evidence that a progressive, home-based physical activity program delivered via DVD is effective in maintaining increased levels of physical activity in younger members of the older adult population. It underscores the importance of studying long-term maintenance of intervention results and of examining subgroups within this population. Finally, it illustrates a need to develop methods to promote maintenance of activity in older members of the population when using home-based designs.

\section{Acknowledgments}

The authors express their sincere appreciation to Susan Houseworth, project coordinator, for this study. They would also like to thank Bill Yauch and RiellyBoy Productions for DVD production; Erica Urrego, our DVD exercise leader; and Grant Henry, Lynda Matejkowski, Joyce O’Donnell, Bernard Puglisi, Paula Smith, and Peter Tan, our DVD exercise models. Finally, the authors extend thanks to Andrew Weil, MD, for the generous contribution of the Healthy Aging DVDs.

\section{Funding Source and Trial Registration}

This work was supported by the National Institute on Aging at the National Institutes of Health (Grant Number 2R01 AG20118). It is registered at www.clinicaltrials.gov (No. NCT01030419).

\section{References}

1. Rowe JW, Kahn RL. Successful Aging. Gerontologist. 1997; 37(4):433-440. DOI: 10.1093/geront/ 37.4.433 [PubMed: 9279031]

2. Depp CA, Jeste DV. Definitions and predictors of successful aging: a comprehensive review of larger quantitative studies. Am J Geriatr Psychiatry. 2006; 14(1):6-20. DOI: 10.1097/01.JGP. 0000192501.03069.bc [PubMed: 16407577] 
3. Warburton DER, Nicol CW, Bredin SSD. Health benefits of physical activity: The evidence. Can Med Assoc J. 2006; 174(6):801-809. DOI: 10.1503/cmaj.051351 [PubMed: 16534088]

4. Miller ME, Rejeski WJ, Reboussin BA, Ten Have TR, Ettinger WH. Physical activity, functional limitations, and disability in older adults. J Am Geriatr Soc. 2000; 48(10):1264-1272. [PubMed: 11037014]

5. Wójcicki TR, Fanning J, Awick EA, Olson EA, Motl RW, McAuley E. Maintenance effects of a DVD-delivered exercise intervention on physical function in older adults. J Gerontol A Biol Sci Med Sci. 2014; :glu188.doi: 10.1093/gerona/glu188

6. World Health Organization. Global Recommendations on Physical Activity for Health. 2010. http:// whqlibdoc.who.int/publications/2010/9789241599979_eng.pdf

7. The Centers for Disease Control and Prevention. Behavioral Risk Factor Surveillance System Survey Questionnaire. Atlanta, Georgia: U.S.; 2013.

8. Tucker JM, Welk GJ, Beyler NK. Physical activity in U.S. adults: compliance with the Physical Activity Guidelines for Americans. Am J Prev Med. 2011; 40(4):454-461. DOI: 10.1016/j.amepre. 2010.12.016 [PubMed: 21406280]

9. Gell NM, Rosenberg DE, Demiris G, Lacroix AZ, Patel KV. Patterns of technology use among older adults with and without disabilities. Gerontologist. 2013; :gnt166.doi: 10.1093/geront/gnt166

10. McAuley E, Wójcicki TR, White SM, et al. Physical activity, function, and quality of life: design and methods of the FlexToBa trial. Contemp Clin Trials. 2012; 33(1):228-236. DOI: 10.1016/j.cct. 2011.10.002 [PubMed: 22024470]

11. McAuley E, Wójcicki TR, Gothe NP, et al. Effects of a DVD-delivered exercise intervention on physical function in older adults. Journals Gerontol Ser A Biol Sci Med Sci. 2013; 68(9):10761082. DOI: $10.1093 /$ gerona/glt014

12. Gothe NP, Wójcicki TR, Olson Ea, et al. Physical activity levels and patterns in older adults: the influence of a DVD-based exercise program. J Behav Med. 2014; doi: 10.1007/s10865-014-9581-6

13. Mailey EL, Gothe NP, Wójcicki TR, et al. Influence of allowable interruption period on estimates of accelerometer wear time and sedentary time in older adults. J Aging Phys Act. 2014; 22(2): 255-260. DOI: 10.1123/japa.2013-0021 [PubMed: 23752299]

14. Actigraph. What are counts?. 2011. https://help.theactigraph.com/entries/20723176-What-arecounts-. Accessed January 26, 2015.

15. Copeland JL, Esliger DW. Accelerometer assessment of physical activity in active, healthy older adults. J Aging Phys Act. 2009; 17(1):17-30. [PubMed: 19299836]

16. Godin G, Shephard RJ. A simple method to assess exercise behavior in the community. Can J Appl Sport Sci. 1985; 10(3):141-146. [PubMed: 4053261]

17. King AC. Interventions to promote physical activity by older adults. Journals Gerontol Ser A Biol Sci Med Sci. 2001; 56(Supplement 2):36-46. DOI: 10.1093/gerona/56.suppl_2.36

18. Oka RK, King AC, Young DR. Sources of social support as predictors of exercise adherence in women and men ages 50 to 65 years. Womens Health. 1995; 1(2):161-175. [PubMed: 9373378]

19. Glasgow RE, Lichtenstein E, Marcus AC. Why don't we see more translation of health promotion research to practice? rethinking the efficacy-to-effectiveness transition. Am J Public Health. 2003; 93(8):1261-1267. DOI: 10.2105/AJPH.93.8.1261 [PubMed: 12893608]

20. Smith, A. Older Adults and Technology Use: Usage and Adoption. Pew Research Center Internet and the American Life Project; 2014. http://www.pewinternet.org/2014/04/03/older-adults-andtechnology-use/

21. Fjeldsoe BS, Miller YD, Marshall AL. MobileMums: a randomized controlled trial of an SMSbased physical activity intervention. Ann Behav Med. 2010; 39(2):101-111. DOI: 10.1007/ s12160-010-9170-z [PubMed: 20174902]

22. Heisler M, Halasyamani L, Resnicow K, et al. "I am not alone": the feasibility and acceptability of interactive voice response-facilitated telephone peer support among older adults with heart failure. Congest Hear Fail. 2007; 13(3):149-157. DOI: 10.1111/j.1527-5299.2007.06412.x

23. Fanning J, Mullen SP, McAuley E. Increasing physical activity with mobile devices: a metaanalysis. J Med Internet Res. 2012; 14(6):e161.doi: 10.2196/jmir.2171 [PubMed: 23171838]

24. Green CA, Pope CR. Gender, psychosocial factors and the use of medical services: a longitudinal analysis. Soc Sci Med. 1999; 48(10):1363-1372. [PubMed: 10369437] 
25. Handy, S. Does the Built Environment Influence Physical Activity: Examining the Evidence. Transportation Research Board; Washington DC: 2005. 


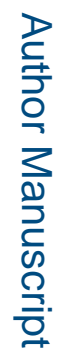

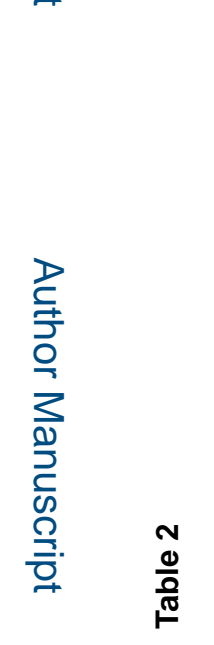

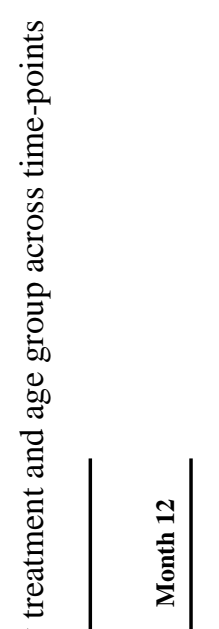

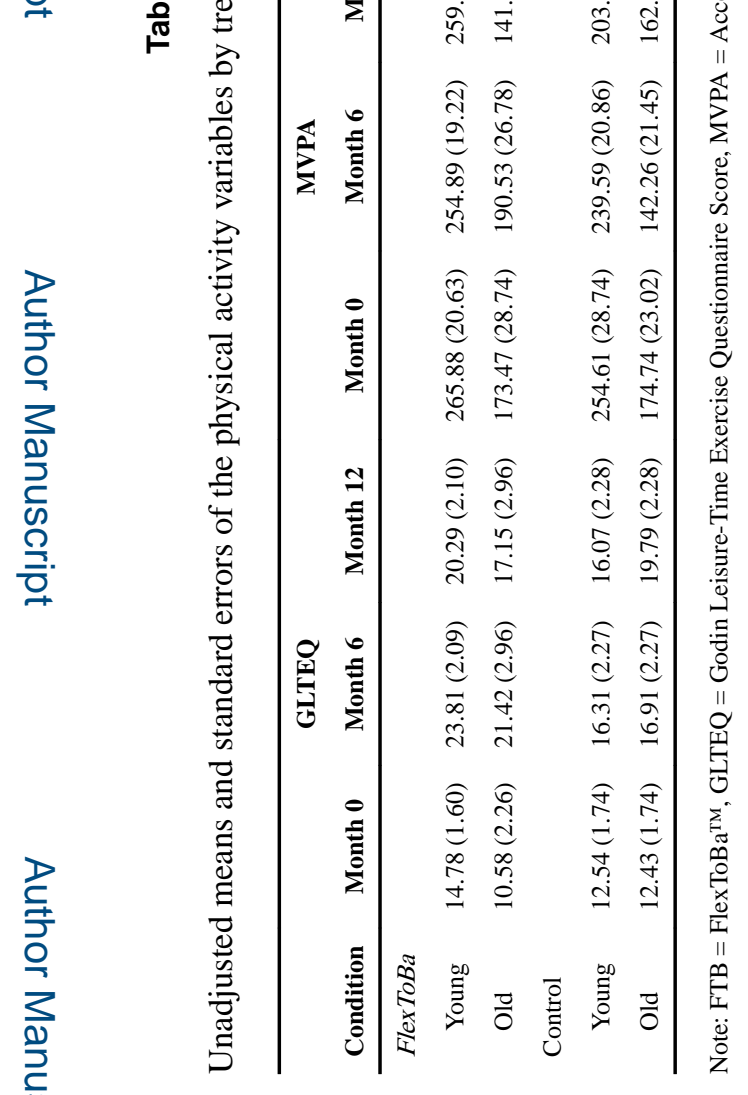

J Phys Act Health. Author manuscript; available in PMC 2017 July 19. 


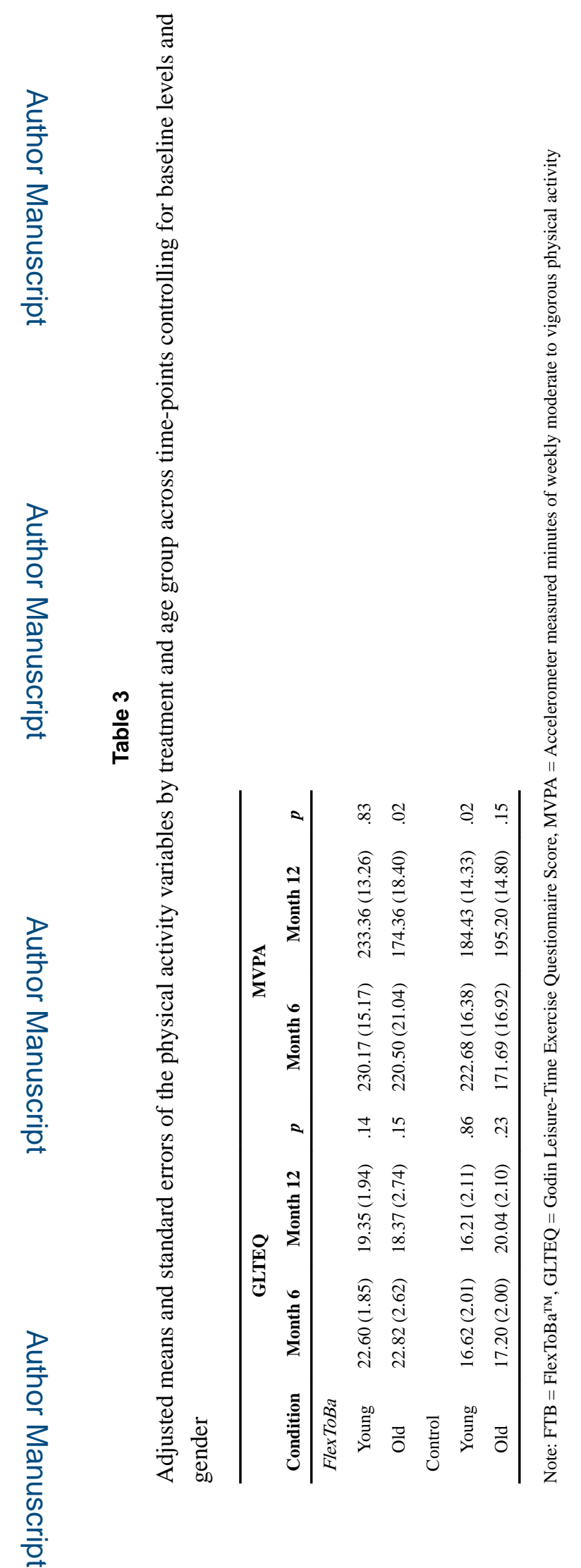

J Phys Act Health. Author manuscript; available in PMC 2017 July 19. 